\title{
Efficient Industrial Control Systems risk assessment using the attack path to the critical device
}

\author{
Mariko Fujimoto \\ Nagoya Institute of Technology \\ Japan \\ m.shigemori.037@stn.nitech.ac.jp \\ Takuho Mitsunaga \\ Toyo University \\ Japan \\ takuho.mitsunaga@iniad.org
}

\author{
Wataru Matsuda* \\ The University of Tokyo \\ Japan \\ wataru.matsuda.ev@hco.ntt.co.jp \\ Yoshihiro Hashimoto \\ Nagoya Institute of Technology \\ Japan \\ hashimoto@nitech.ac.jp
}

\begin{abstract}
In cyberattacks against Industrial Control Systems (ICS), one of the final goals of attackers is to impair ICS operations. Recent ICS tend to be connected with other networks and are often implemented with common technologies in order to increase production efficiency and reduce costs. This leads to the creation of new attack surfaces that increase the cyber risks of these systems. Although preventing system intrusion without fault could be difficult, if attacks can be prevented at an early stage before they reach the physical world, damage can be minimized. Thus, appropriate cybersecurity risk assessment methods for ICS are necessary. ICS risk assessment methods proposed in previous research require more time to administer, and they heavily depend on the analysts' particular expertise and skill to efficiently examine attack scenarios and attack paths. In this research, we propose an efficient quantitative risk assessment method to evaluate the risk of possible attacks on critical ICS devices. This method uses packet capture data in the ICS network to automatically enumerate possible attack paths and calculates the risk level of each attack path. It then comprehensively evaluates the risk level of an entire ICS by utilizing multiple metrics such as the severity of vulnerabilities that exist in specific devices or communication methods, security measures that have been taken, and the features of the attack paths to the critical devices.
\end{abstract}

\section{CCS CONCEPTS}

- Security and privacy $\rightarrow$ Network security.

\section{KEYWORDS}

Industrial Control System, cyber security, risk assessment, attack path

${ }^{*}$ Presently with NTT Secure Platform Laboratories

\section{(c) $(1) \Theta$}

This work is licensed under a Creative Commons Attribution-NoDerivs International 4.0 License.

MSIE 2021, April 2-4, 2021, Osaka, Japan

(C) 2021 Copyright held by the owner/author(s)

ACM ISBN 978-1-4503-8888-7/21/04.

https://doi.org/10.1145/3460824.3460859

\section{ACM Reference Format:}

Mariko Fujimoto, Wataru Matsuda, Takuho Mitsunaga, and Yoshihiro Hashimoto. 2021. Efficient Industrial Control Systems risk assessment using the attack path to the critical device. In $20213 \mathrm{rd}$ International Conference on Management Science and Industrial Engineering (MSIE 2021), April 2-4, 2021, Osaka, Japan. ACM, New York, NY, USA, 7 pages. https: //doi.org/10.1145/3460824.3460859

\section{CYBER RISKS IN INDUSTRIAL CONTROL SYSTEMS}

ICS were considered to possess no cybersecurity risks since they were operated in isolated networks. However, in modern ICS, a variety of common technologies are used, and multiple systems are oftentimes interconnected. The use of common technologies makes attack activities easier since an abundance of information and tools are openly available. If an ICS network is connected to another network, there is also the security risk of intrusion into the ICS network through connected adjacent networks.

The Cyber Kill Chain is a part of the Intelligence-Driven Defense model for the identification and prevention of cyber intrusion activities. According to the Cyber Kill Chain of ICS, [2], defined by the SANS Institute, attack activities are divided into attacks against IT systems and attacks against ICS. For example, attackers first intrude into an employee's PC, then expand into the ICS network through a Gateway server, etc. Attackers who make their way into the ICS network tries to compromise ICS devices. Controllers and PLCs are typical targets of attacks [16] [17], thus attackers tend to expand infections to devices that can directly operate controllers or PLCs. The following $(1.1,1.2,1.3)$ are typical methods to expand the intrusion scope and to operate the ICS maliciously.

\subsection{Leveraging vulnerabilities}

Attackers leverage vulnerabilities for expanding infections, gaining higher privilege, executing malicious code, etc. The impact of a vulnerability is different depending on the type, and one of the most serious vulnerability types is the remote code execution type, which allows arbitrary code execution from remote hosts.

\subsection{Leveraging legitimate functions}

Attackers sometimes leverage legitimate functions to expand infections. For instance, Pass the hash[8] and Golden Ticket[15] are 
two famous attack methods leveraging legitimate accounts of the Windows operating system.

\subsection{Leveraging vulnerable communications}

Some ICS devices including PLCs use vulnerable communication methods that are not protected with encryption or authentication, and they are vulnerable to replay attacks [17]. A replay attack in this paper is defined as an attack where attackers snoop data transmitted among legitimate ICS devices and then use this data to send unauthorized ICS commands. For instance, attackers can snoop communications between HMIs and PLCs and then send unauthorized commands to PLCs.

\section{RELATED RESEARCH}

This chapter describes related research on risk assessment methods for analyzing the risk of cyber attacks using attack trees or attack paths that are easy to automate. [7] proposes a method to analyze attack patterns using attack trees. [3] proposes the application of attack trees to an ICS environment. The purpose of research [7][3] is to identify corresponding security measures, thus they are different from the goal of our research. [12] proposes a method to consider appropriate security measures for ICS based on attack paths, but it does not focus on ICS specific security risks.

Some research propose methods to analyze the threat of attacks using attack paths based on system architectures. [11] proposes a method for creating attack paths based on vulnerabilities, network information, account information, etc. [1] extends the research [11] to other network attacks such as ARP spoofing and route hijacking. [6] proposes an efficient method for creating attack paths for large networks based on vulnerabilities and network information. They focus on specific attack methods against generic IT systems and do not focus on attacks against ICS.

[13] proposes a risk assessment method for ICS considering multiple aspects such as attacks leveraging legitimate functions in addition to vulnerabilities. It is a comprehensive risk assessment method, but it takes a lot of time and depends on the analyst's skill since it creates attack paths based on each attack scenario analyzed by an analyst. We refer to [13], then propose a more efficient risk assessment method.

\section{PROPOSED METHOD}

We propose an efficient quantitative risk assessment method to evaluate the risk of attacks reaching critical ICS devices. Our method calculates the risk level of attack reachability to the critical devices from a specific computer (e.g. a computer that a security alert is detected). Critical devices indicate the most important ICS devices that need to be protected, and they should be defined in each organization. In this research, we choose PLCs as critical devices. The contribution of our research is as follows.

- The proposed method considers cyber risks specific to ICS and can evaluate the cyber risk level mechanically without depending on human skill.

- The proposed method can reduce the time cost of risk assessment since it analyzes communication traffic to automatically generate possible attack paths, and iterates through each of the paths.

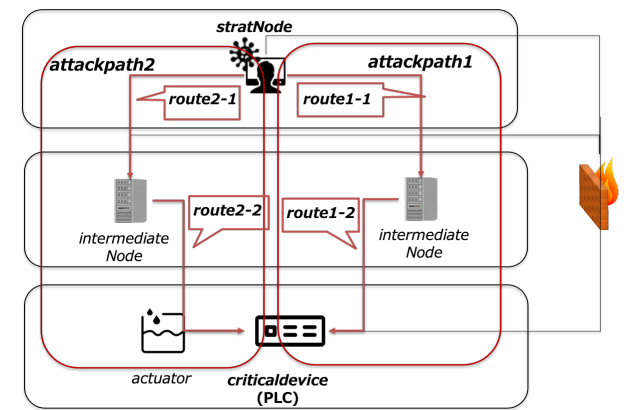

Figure 1: Conceptual diagram of the attack paths

- The proposed method can identify an attack path to a critical device with the highest risk factor and can simulate the effectiveness of implementing security measures.

\subsection{Hypotheses}

We assume that a successful attack depends on the following elements.

- Vulnerabilities that exist on each host

- The number of remote access services available

- The number of vulnerable communications among hosts

- Security measures taken on each computer and network

\subsection{Enumeration method of the attack path}

All possible network attack paths should first be enumerated. Figure 1 is a conceptual diagram that shows an example of possible attack paths. startNode is an arbitrary computer where the attack paths initiate. This is oftentimes a compromised computer where a security alert is detected. intermediateNode are devices that exist on the route between the startNode and the critical devices. criticaldevice is the most important ICS device that needs to be protected. The proposed method enumerates all paths that can reach from startNode to criticaldevice by identifying network reachability by using passive network scanning among hosts.

(1) Extract all destination hosts for the specified host from packets. This step should start from startNode.

(2) Repeat step 1 for every host until criticaldevice is reached.

For efficient automated risk assessment, the proposed method narrows down attack activities to unauthorized manipulation and DoS attacks against ICS devices, since they lead to critical physical damages of ICSs. For criticaldevice, the proposed method assesses attack activities that could affect the behavior of the criticaldevice. In the case of intermediateNode, since these hosts can be leveraged as stepping stones to criticaldevice, the proposed method mainly assesses attack activities that allow the expansion of infections.

\subsection{Risk assessment metrics}

3.3.1 Vulnerabilities. Vulnerabilities that exist on each host are one of the metrics of assessing possibilities of successful attacks. Since analyzing all existing vulnerabilities could be very costly, we narrow down the vulnerabilities that are analyzed to the ones below. 
Table 1: Metrics for vulnerabilities

\begin{tabular}{|l|l|l|l|l|}
\hline Score & CVSS3 metrics & Value & Critical device & Intermediate node \\
\hline 1 & Attack Complexity & Low & Evaluate & Evaluate \\
\hline 1 & Privileges Required & None & Evaluate & Evaluate \\
\hline 1 & User Interaction & None & Evaluate & Evaluate \\
\hline 1 & Integrity Impact & High & Evaluate & - \\
\hline 1 & Availability Impact & High & Evaluate & - \\
\hline
\end{tabular}

Table 2: Metrics for remote access services

\begin{tabular}{|l|l|l|}
\hline Score & Service & Description \\
\hline 1 & Secure Shell (SSH) & $\begin{array}{l}\text { A cryptographic protocol generally used to access Unix-like } \\
\text { operating systems }\end{array}$ \\
\hline 2 & Telnet & $\begin{array}{l}\text { A protocol generally used to access Unix-like operating systems. } \\
\text { Data does not encrypt by default. }\end{array}$ \\
\hline 2 & $\begin{array}{l}\text { Remote } \\
\text { (rsh)/rlogin }\end{array}$ & $\begin{array}{l}\text { A protocol used to execute remote shell or access remote com- } \\
\text { puters. Data does not encrypted. }\end{array}$ \\
\hline 2 & $\begin{array}{l}\text { Server Message Block } \\
\text { (SMB) }\end{array}$ & $\begin{array}{l}\text { A protocol generally used to access Windows system. Since } \\
\text { there are common attack methods such as Pass the hash, the } \\
\text { score is set to 2. }\end{array}$ \\
\hline 1 & $\begin{array}{l}\text { Remote Desktop Proto- } \\
\text { col (RDP) }\end{array}$ & $\begin{array}{l}\text { A cryptographic protocol generally used to access the Windows } \\
\text { system using GUI }\end{array}$ \\
\hline 2 & Kerberos & $\begin{array}{l}\text { A cryptographic protocol generally used in Active Directory. } \\
\text { Since there are common attack methods such as Golden Tickets, } \\
\text { the score is set to 2. }\end{array}$ \\
\hline
\end{tabular}

Table 3: Metrics for communications

\begin{tabular}{|l|l|l|}
\hline Score & Metrics & Description \\
\hline 1 & Encryption & None \\
\hline 1 & Authentication & None \\
\hline 1 & Protocol & Standard protocol \\
\hline
\end{tabular}

- Vulnerabilities on criticaldevice: Vulnerabilities that affect integrity and availability.

- Vulnerabilities on intermediateNode: Vulnerabilities that allow arbitrary code execution remotely (Remote Code Execution vulnerabilities).

Furthermore, the proposed method narrows down the analyzed vulnerabilities to those where the difficulty level of leveraging them is rather low. The existence of Proof Of Concepts and CVSS3 metrics [9] are used to decide on the difficulty level of each vulnerability. The procedure is as follows.

(1) Pick up vulnerabilities where Proof Of Concepts (exploit code) are published on the Internet. The proposed method searches for Proof Of Concepts of each vulnerability from the Exploit Database [14] and reference URLs published in the NATIONAL VULNERABILITY DATABASE [10].

(2) Pick up remotely exploitable vulnerabilities where the CVSS3 metric of "Attack Vector" is "Network" or "Adjoin".

(3) Add score to vulnerabilities that meet conditions shown in Table 1.
3.3.2 Available remote access services. In addition to exploitable vulnerabilities, a successful attack also depends on the number of remote access services available. For efficient analysis, only remote access services that allow remote login to the system is considered as part of the proposed method. The metrics indicate the possibility of whether intermediateNode could be exploited as a steppingstone. Table 2 lists remote access services and their risk score.

3.3.3 Vulnerable communications. Table 3 shows the metrics for vulnerable communication. A "Standard protocol" is defined as a protocol, including common ICS protocols, that meet the following conditions.

- The protocol uses Internet standard protocols such as TCP, UDP, IP, etc.

- The specification of the protocol is published.

3.3.4 Asset priority. The asset priority metric indicates how much potential damage there could be if the specific asset is compromised. It is based on what functions the asset is responsible for in the ICS and is also dependent on what kind of attack activities could be initiated on the asset. The asset priority level is shown in Table 4.

3.3.5 Security level. A successful attack also depends on security measures taken on each computer and network. The security level is calculated based on the security measures taken on the source host and destination host in each attack route.

The proposed method selected security measures shown in Table 5 from the ICS security guidelines[5] and incorporated them in as 
Table 4: Asset priority level

\begin{tabular}{|l|l|l|}
\hline Score & Description & Examples \\
\hline 3 & $\begin{array}{l}\text { Devices that can change the physical state of an } \\
\text { ICS }\end{array}$ & OPC Server, HMI, Engineering Workstation \\
\hline 2 & $\begin{array}{l}\text { Devices that can retreive ICS data but cannot } \\
\text { change data }\end{array}$ & Read only OPC Server and HMI, historian \\
\hline 1 & Other ICS related devices & Production control server \\
\hline 0 & Other devices & Business PC, Web server \\
\hline
\end{tabular}

Table 5: Security level

\begin{tabular}{|l|l|}
\hline Score & Security measure \\
\hline 1 & Anti virus software \\
\hline 1 & $\begin{array}{l}\text { Password is more than 8 characters and includes upper and lowercase letters, numbers, and } \\
\text { special characters. A unique password is set on each host }\end{array}$ \\
\hline 1 & Minimize account privileges \\
\hline 1 & $\begin{array}{l}\text { Memory protection (e.g. LSA protection, Credential Guard: Windows memory protection } \\
\text { methods against unauthorized access to credentials) }\end{array}$ \\
\hline 1 & Encryption and access control against ICS data \\
\hline 1 & Authentication and authentication of ICS application \\
\hline 1 & Application whitelist \\
\hline 1 & Appropriate encryption key management \\
\hline 1 & Network security solution (e.g. ICS firewall, IPS ) \\
\hline
\end{tabular}

metrics. If the security measures defined in Table 5 are taken, the corresponding score is subtracted from the risk level.

\subsection{Procedure for calculating the risk level}

Figure 2 shows an example of calculating the risk level. (1) is the formula to calculate the total risk level of each attack $\operatorname{path}($ attackpathx $)$.

$$
\text { risklevel }_{\text {pathX }}=\prod_{i=\text { start Node }}^{\text {critical }} \text { risklevel }_{i}
$$

The risk level of the entire system is defined as (2), and is the highest risk level of all the attack path risk levels.

$$
\max \left\{\text { risklevel }_{\text {pathX }}, X=1,2, \cdots, n\right\}
$$

The risk level of each attack path $\left(\right.$ risklevel $\left._{\text {pathX }}\right)$ is the product of the risk level of each network route ( risklevel $_{i}$ ) on the attack path. Related research [13] defined that the risk level of each attack path is the minimum value of the risk level of each network route. However, our evaluation results showed that that method does not properly evaluate the risk levels under some conditions.

(3) shows the formula for calculating the risk level of each route.

$$
\begin{array}{r}
\text { risklevel }_{i}=\left(\text { threatlevel }_{d}+\text { prioritylevel }_{s}-\right. \\
\left.\left(\text { securitylevel }_{s}+\text { securitylevel }_{d}\right)\right) / M A X R I S K L E V E L
\end{array}
$$

The risk level of each route is calculated using the threat level

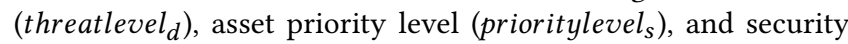
level(securitylevel , securitylevel $_{d}$ ).

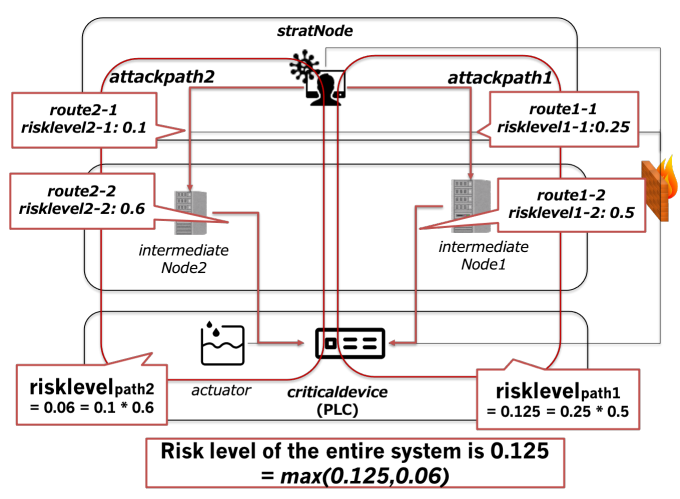

Figure 2: Example of calculating risk level

3.4.1 Threat level. The threat level is calculated as (4).

threatlevel $=$ vullevel $_{d}+$ remoteservicecht + vulcomcnt

vullevel $_{d}$ is the risk level that indicates the existence of vulnerabilities on the destination host in each attack route. If the destination host has vulnerabilities that meet the conditions defined in section 3.3.1, the corresponding score is added. The maximum vullevel $l_{d}$ is 3. If the vullevel exceeds 3 , vullevel $_{d}$ is set to 3 .

remoteservicecnt is the accessible remote access services of the destination host in each attack route. If the destination host has 


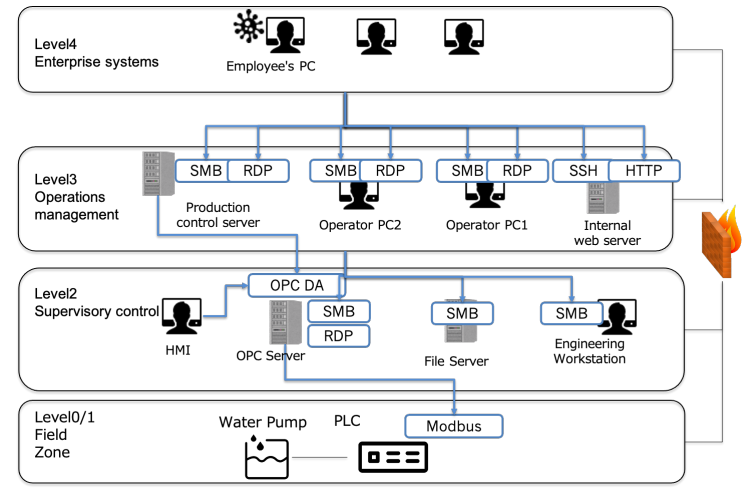

Figure 3: ICS testbed for evaluation

accessible remote access services defined in Table 2, the corresponding risk level is added per remote access service. The maximum remoteservicecnt is 3 . If the remoteservicecnt exceeds 3 , remoteservicecnt is set to 3 .

vulcomcnt is the vulnerable communications between the source host and destination host in each attack route. If vulnerable communications that meet any conditions in Table 3 exist, the risk level is added per condition. The maximum vulcoment is 3 . If the vulcomcnt exceeds 3 , vulcomcnt is set to 3 .

3.4.2 Asset priority level. The asset priority level(prioritylevel s $_{\text {) }}$ indicates the damage level of each computer on an attack path if it is compromised. Thus it is evaluated with the asset priority level of the source host in each attack route.

3.4.3 Security level. The security level(securitylevel , securitylevel $_{d}$ ) is the security measures taken on the source host and destination host in each attack route. If the security measures defined in Table 5 are taken, the corresponding risk level is subtracted per security measure. The maximum-security level(securitylevel ${ }_{s}+$ securitylevel $\left._{d}\right)$ is 3 . If the security level exceeds 3 , it is set to 3 .

3.4.4 Normalizing and scoring. The maximum score of vullevel ${ }_{d}$, remoteservicecnt, vulcomcnt and security level(securitylevel s $_{s}+$ securitylevel $_{d}$ ) are adjustable. In this paper, we define the maximum score based on previous research[13]. MAXRISKLEVEL is the maximum risk level that is allowed per attack route. To normalize the risk level between 0 to 1 , the risk level is divided by MAXRISKLEVEL.

\section{EVALUATION OF THE PROPOSED METHOD}

We compare the result (successful or failure) of an attack in the penetration test with the risk level of each attack path calculated by the proposed method. For the evaluation, we use an ICS testbed that operates on actual machinery. The testbed is a water pump system that provides water to consumers with flow control and temperature control (Figure 3 and Table 6). It is divided into several network segments based on the reference architecture in IEC 62443[4].

We evaluate whether the proposed method can simulate the risk level of each attack path and the effectiveness of security measures correctly upon the following patterns.

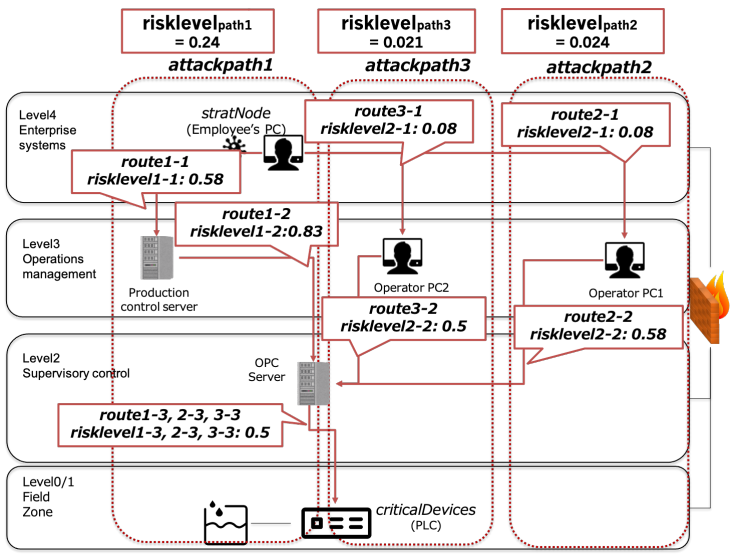

Figure 4: Evaluation result(Without security measures)

\subsection{Evaluation without security measures}

Almost all security measures shown in Table 5 are disabled on many computers, and ICS communications meet the condition in Table 3.

\subsection{Evaluation with security measures}

We place security measures on networks and devices that exist on the attack path with the highest risk factor as calculated in evaluation pattern 1 , then evaluate whether the proposed method can rate the effect of security measures correctly.

\subsection{Evaluation result}

We compared the result of the penetration test with the risk level of each attack path calculated by the proposed method shown in Table 7.

4.3.1 Evaluation result (Without security measures). Table 8 shows the result of evaluation pattern 1 (4.1) that places no security measures. The "Attack result" column indicates whether pentesters were able to attack critical devices (PLCs). As a result, the proposed method found 3 possible attack paths (Figure 4) and rated attackpath 1 as the path with the highest risk factor. This attackpath 1 was the only path where pen-testers successfully attacked the critical devices.

4.3.2 Evaluation result (With security measures). Table 9 shows the evaluation result of evaluation pattern 2 that place security measures (4.2). We placed the following security measures on the networks and devices that existed on the network route route $1-2$ of attackpath 1 with the highest risk level. As a result, the risk level of attackpath 1 decreased to "0.083", and pen-testers were not able to attack the critical devices.

- Close unnecessary ports (RDP and SMB) in route $1-2$.

- Protect the memory of the Production control server and OPC server with LSA protection ${ }^{1}$.

- Set password to the OPC server application.

- Set a unique and strong password to the Production control server.

${ }^{1}$ Additional protection for the Local Security Authority (LSA) process to prevent Code injection that could compromise credentials 
Table 6: Evaluation environment

\begin{tabular}{|l|l|l|}
\hline Device & OS(Level) & Used protocols \\
\hline Water pump & - & - \\
\hline PLC & - & Modbus/TCP \\
\hline OPC Server & Windows Server 2016 & OPC DA/Modbus(TCP) \\
\hline HMI & Windows 10 & OPC DA \\
\hline Engineering workstation & Windows 7 & SMB,Modbus/TCP \\
\hline ICS file server & Windows Server 2016 & SMB \\
\hline Production control server & Windows 7 & OPC DA,RDP,SMB \\
\hline Operator's PC1 & Windows 10 & RDP,SMB \\
\hline Operator's PC2 & Windows 10 & RDP,SMB \\
\hline Internal web server & CentOS 7 & SSH,HTTP \\
\hline Employee's PC & Windows 7/MacOS & SMB,RDP \\
\hline
\end{tabular}

Table 7: Risk assessment metrics

\begin{tabular}{|l|l|l|l|}
\hline & \multicolumn{3}{|c|}{ Risk level } \\
\hline Device & Asset priority & Vulnerability & Security \\
\hline PLC & 3 & 0 & 0 \\
\hline OPC Server & 3 & 3 & $0\left(2^{*}\right)$ \\
\hline HMI & 3 & 3 & 0 \\
\hline Engineering workstation & 3 & 3 & 0 \\
\hline ICS file server & 1 & 3 & 1 \\
\hline Production control server & 1 & 3 & $0\left(2^{*}\right)$ \\
\hline Operator's PC1 & 0 & 0 & 2 \\
\hline Operator's PC2 & 0 & 0 & 3 \\
\hline Internal web server & 0 & 2 & 0 \\
\hline Employee's PC & 0 & 0 & 2 \\
\hline
\end{tabular}

*Value in the bracket means the security level upon the evaluation with security measures.

Table 8: Evaluation result(Without security measures)

\begin{tabular}{|l|l|l|l|l|l|}
\hline Path & $\begin{array}{l}\text { riskLevel } \\
\text { (total) }\end{array}$ & $\begin{array}{l}\text { riskLevel } \\
\text { (route1) }\end{array}$ & $\begin{array}{l}\text { riskLevel } \\
\text { (route2) }\end{array}$ & $\begin{array}{l}\text { riskLevel } \\
\text { (route3) }\end{array}$ & $\begin{array}{l}\text { Attack } \\
\text { result }\end{array}$ \\
\hline 1 & $0.24(5)$ & 0.58 & 0.83 & 0.50 & success \\
\hline 2 & $0.024(1)$ & 0.080 & 0.58 & 0.50 & fail \\
\hline 3 & $0.021(1)$ & 0.083 & 0.50 & 0.50 & fail \\
\hline
\end{tabular}

Table 9: Evaluation result(With security measures)

\begin{tabular}{|l|l|l|l|l|l|}
\hline Path & $\begin{array}{l}\text { riskLevel } \\
\text { (total) }\end{array}$ & $\begin{array}{l}\text { riskLevel } \\
\text { (route1) }\end{array}$ & $\begin{array}{l}\text { riskLevel } \\
\text { (route2) }\end{array}$ & $\begin{array}{l}\text { riskLevel } \\
\text { (route3) }\end{array}$ & $\begin{array}{l}\text { Attack } \\
\text { result }\end{array}$ \\
\hline 1 & $\mathbf{0 . 0 8 3}(4)$ & 0.50 & 0.33 & 0.50 & fail \\
\hline 2 & $0.020(1)$ & 0.080 & 0.50 & 0.50 & fail \\
\hline 3 & $0.021(1)$ & 0.083 & 0.5 & 0.50 & fail \\
\hline
\end{tabular}

Compared with the previous research[13], the proposed method judged the same path with the previous research as a critical path. However, when applying the security measures and the attack failed, the risk level of the critical path decreased by only $20 \%$ while the risk level of the proposed method decreased by $67 \%$. It can be said that the proposed method can calculate the risk level more accurately.

\section{CONCLUSION}

In this research, we proposed an efficient cybersecurity risk assessment method for ICS. The proposed method can be utilized for 
identifying devices on the high-risk network path or simulating the efficiency of implementing security measures. Furthermore, the proposed method can evaluate the cyber risk level automatically and mechanically without depending on human skill. The method is tested on a small testbed as an example. However, The proposed method can extend to a wider class of environments by using automated assessment. In this paper, we used metrics considered in previous research to define the maximum score of metrics used for calculating the risk level. For future work, we will evaluate more ICS operations and attack patterns, and adjust each score based on the evaluation results. In addition, we will further evaluate how certain security measures affect different threats, and how they should be scored.

\section{REFERENCES}

[1] Jaime C Acosta, Edgar Padilla, and John Homer. 2016. Augmenting attack graphs to represent data link and network layer vulnerabilities. In MILCOM 2016-2016 IEEE Military Communications Conference. IEEE, 1010-1015.

[2] Michael J. Assante and Robert M. Lee. 2015. The Industrial Control System Cyber Kill Chain,. https://www.sans.org/reading-room/whitepapers/ICS/paper/36297

[3] Eric J Byres, Matthew Franz, and Darrin Miller. 2004. The use of attack trees in assessing vulnerabilities in SCADA systems. In Proceedings of the international infrastructure survivability workshop. Citeseer, 3-10.

[4] The International Electrotechnical Commission. [n.d.]. IEC/TS 62443-1-1 Industrial communication networks â $€$ " Network and system security.

[5] Cybersecurity and Infrastructure Security Agency (CISA). [n.d.]. Recommended Practices. https://www.us-cert.gov/ics/Recommended-Practices
[6] Heng Li, Yongjun Wang, and Yuan Cao. 2017. Searching forward complete attack graph generation algorithm based on hypergraph partitioning. Procedia Computer Science 107 (2017), 27-38.

[7] Andrew P Moore, Robert J Ellison, and Richard C Linger. 2001. Attack modeling for information security and survivability. Technical Report. Carnegie-Mellon Univ Pittsburgh Pa Software Engineering Inst.

[8] Jeffrey A Nichols, Benjamin A Taylor, and Laura Curtis. 2016. Security resilience: Exploring windows domain-level defenses against post-exploitation authentication attacks. In Proceedings of the 11th Annual Cyber and Information Security Research Conference. 1-4.

[9] National Vulnerability Database (NVD). [n.d.]. Common Vulnerability Scoring System Calculator. https://nvd.nist.gov/vuln-metrics/cvss/v3-calculator

[10] The Department of Homeland Security. [n.d.]. NATIONAL VULNERABILITY DATABASE. https://nvd.nist.gov/vuln

[11] Xinming Ou, Sudhakar Govindavajhala, and Andrew W Appel. 2005. MulVAL: A Logic-based Network Security Analyzer.. In USENIX security symposium, Vol. 8. Baltimore, MD, 113-128.

[12] Nayot Poolsappasit, Rinku Dewri, and Indrajit Ray. 2011. Dynamic security risk management using bayesian attack graphs. IEEE Transactions on Dependable and Secure Computing 9, 1 (2011), 61-74.

[13] Masaki Inokuchi Tomohiko Yagyu Ryo Mizushima, Hirofumi Ueda. 2020. Consideration of Calculation of Evaluation Indicies for Cyber Attack Risk Assessment(Japanese). Symposium on Cryptography and Information Security (2020).

[14] Offensive Security. [n.d.]. Exploit Database. https://www.exploit-db.com/

[15] Benjamin Delp Skip Duckwall. 2014. Abusing Kerberos. https://www.blackhat.com/docs/us-14/materials/us-14-Duckwall-AbusingMicrosoft-Kerberos-Sorry-You-Guys-Don't-Get-It-wp.pdf

[16] Hitomi Takagi, Takahito Morita, Masafumi Matta, Hiroki Moritani, Takashi Hamaguchi, Sun Jing, Ichiro Koshijima, and Yoshihiro Hashimoto. 2015. Strategic security protection for industrial control systems. In 2015 54th Annual Conference of the Society of Instrument and Control Engineers of Japan (SICE). IEEE, 986-992.

[17] Haroon Wardak, Sami Zhioua, and Ahmad Almulhem. 2016. PLC access control: a security analysis. In 2016 World Congress on Industrial Control Systems Security (WCICSS). IEEE, 1-6. 